\title{
Elongation of Triplet Lifetime Caused by Intramolecular Energy Hopping in Diphenylanthracene Dyads Oriented to Undergo Efficient Triplet-Triplet Annihilation Upconversion
}

\author{
Masaya Kanoh, ${ }^{1}$ Yasunori Matsui,,${ }^{1,2, *}$ Kiyomasa Honda, ${ }^{1}$ Yuto Kokita, ${ }^{1}$ \\ Takuya Ogaki, ${ }^{1,2}$ Eisuke Ohta, ${ }^{1,2}$ and Hiroshi Ikeda ${ }^{1,2, *}$ \\ ${ }^{1}$ Department of Applied Chemistry, Graduate School of Engineering, Osaka Prefecture University, 1-1 Gakuen-cho, \\ Nakaku, Sakai, Osaka 599-8531, Japan \\ ${ }^{2}$ The Research Institute for Molecular Electronic Devices (RIMED), Osaka Prefecture University, 1-1 Gakuen-cho, Nakaku, \\ Sakai, Osaka 599-8531, Japan \\ E-mail: matsui@chem.osakafu-u.ac.jp; ikeda@chem.osakafu-u.ac.jp
}

\section{Table of Contents}

1. General

2. Preparation of Substrates

S1

Preparation of Substrates

3. Phosphorescence Quenching Experiments $\quad$ S3

4. Raw Data of UC Luminescence Spectra $\quad$ S4

5. Determination of Triplet Lifetime under the Benzophenone-sensitization Conditions S4

6. NMR Spectra $\quad$ S5

$\begin{array}{ll}\text { 7. Theoretical Calculations } & \text { S9 }\end{array}$

\section{General}

Melting points (mp) were obtained with a Yanaco MP-500 or differential scanning calorimetry (DSC) system a Rigaku Thermo plus $2 \mathrm{EVO}$ and are reported uncorrected. ${ }^{1} \mathrm{H}$ and ${ }^{13} \mathrm{C}$ NMR spectra were recorded at 400 and $100 \mathrm{MHz}$, respectively, on a Bruker AVANCE NEO 400 spectrometer. Chemical shifts $(\delta)$ are reported in ppm using the signal of tetramethylsilane $(0.00 \mathrm{ppm})$ and $\mathrm{CDCl}_{3}(77.0 \mathrm{ppm})$ as an internal standard for ${ }^{1} \mathrm{H}$ and ${ }^{13} \mathrm{C} \mathrm{NMR}$, respectively. IR spectra were recorded on a JASCO FT/IR 8300 spectrometer with attenuated total reflection (ATR) method. Low-resolution (LR) atmospheric solid analysis probe (ASAP) mass measurements were carried out with a Shimadzu LCMS-2020 and LabSolutions LCMS software. LR fast atom bombardment (FAB)-mass spectra were recorded with a JEOL JMS-700 spectrometer in a positive mode with 3-nitrobenzyl alcohol (NBA) as a matrix. High-resolution (HR) electrospray ionization (ESI) mass measurements were carried out on a Bruker micrOTOF system after calibration using a sodium formate solution. Elemental analyses were carried out at the Research and Analytical Centre at Kanazawa University. UV-Vis absorption and photoluminescence spectra were recorded on JASCO V-570 and FP-8500 spectrophotometers, respectively. Absolute fluorescence quantum yields were determined by utilizing the integrating sphere method with a HAMAMATSU PHOTONICS C9920-02 absolute photoluminescence quantum yields measurement system. Fluorescence lifetime (FL) values were determined by using a HORIBA Jobin Yvon FluoroCube lifetime spectrofluorometer and analyzed using DAS6 FL decay analysis software. 


\section{Preparation of Substrates}

(a)

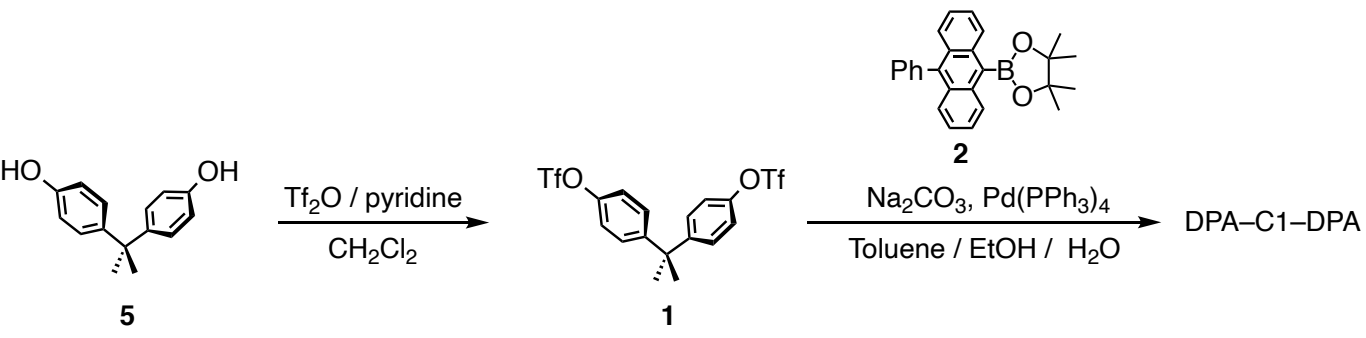

(b)
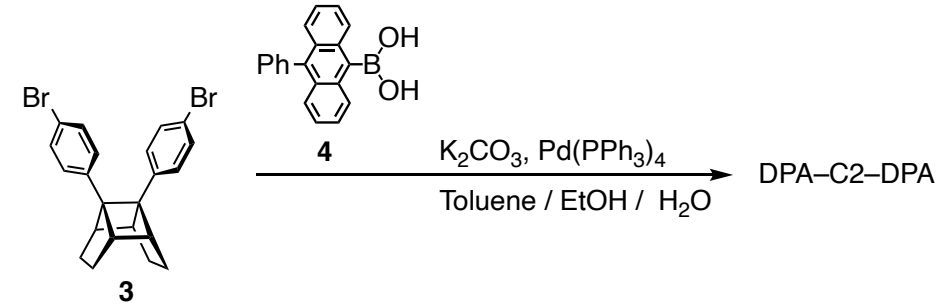

Scheme S1. Routes for synthesis of (a) DPA-C1-DPA and (b) DPA-C2-DPA.

2,2-Bis(4-(trifluoromethyl)sulfonyloxyphenyl)propane (1): In a $200 \mathrm{~mL}$ three-necked flask, bisphenol A (5, $2.82 \mathrm{~g}, 10$ mmol), pyridine $(2.4 \mathrm{~mL}, 10 \mathrm{mmol})$, and dry $\mathrm{CH}_{2} \mathrm{Cl}_{2}(50 \mathrm{~mL})$ were placed under argon. Trifluoromethanesulfonic anhydride (5.0 $\mathrm{mL}, 30 \mathrm{mmol}$, in $10 \mathrm{~mL}$ of $\mathrm{CH}_{2} \mathrm{Cl}_{2}$ ) was added dropwise to the flask over $30 \mathrm{~min}$ at $0{ }^{\circ} \mathrm{C}$, and the solution was stirred at room temperature. After stirring for $16 \mathrm{~h}$, the mixture was quenched by addition of water $(50 \mathrm{~mL})$ at $0{ }^{\circ} \mathrm{C}$ and extracted with $\mathrm{CHCl}_{3}(50 \mathrm{~mL} \times 3)$. The combined organic extracts were washed with brine, dried over anhydrous $\mathrm{Na}_{2} \mathrm{SO}_{4}$, and evaporated to dryness under reduced pressure. The residual brown oil was subjected to column chromatography on $\mathrm{SiO}_{2}\left(\mathrm{CH}_{2} \mathrm{Cl}_{2}: n\right.$ hexane $=1: 1)$. Recrystallization from $n$-hexane afforded 1 as colorless plates $(4.79 \mathrm{~g}, 9.7 \mathrm{mmol})$ in $97 \%$ yield. mp $58-58.5$ ${ }^{\circ} \mathrm{C} ;{ }^{1} \mathrm{H}$ NMR $\left(400 \mathrm{MHz}, \mathrm{CDCl}_{3}\right) \delta_{\mathrm{ppm}} 1.69$ (s, 6H), 7.17 (AA'BB', $\left.J=6.8,2.0 \mathrm{~Hz}, 4 \mathrm{H}\right), 7.28$ (AA'BB', $\left.J=6.8,2.0 \mathrm{~Hz}, 4 \mathrm{H}\right)$; ${ }^{13} \mathrm{C}$ NMR $\left(100 \mathrm{MHz}, \mathrm{CDCl}_{3}\right) \delta_{\mathrm{ppm}} 30.7$ (2C), 42.9, 118.7 (q, $\left.J_{\mathrm{C}-\mathrm{F}}=318 \mathrm{~Hz}, 2 \mathrm{C}\right), 121.0(4 \mathrm{C}), 128.6(4 \mathrm{C}), 147.8(2 \mathrm{C}), 150.2$ (2C); IR (ATR) $v=705,742,760,784,806,844,889,1016,1140,1200,1216,1249,1417,1428,1417,1428,1501 \mathrm{~cm}^{-1}$; LR-mass (ASAP) $m / z=493[\mathrm{M}+\mathrm{H}]^{+}$for $\mathrm{C}_{17} \mathrm{H}_{14} \mathrm{~F}_{6} \mathrm{O}_{6} \mathrm{~S}_{2}$

2,2-Bis(4-(10-phenylanthracen-9-yl)phenyl)propane (DPA-C1-DPA): In a $100 \mathrm{~mL}$ three-necked flask, 1 (0.344 g, 0.7 mmol), 4,4,5,5-tetramethyl-2-(10-phenylanthracen-9-yl)-1,3,2-dioxaborolane $\quad(2, \quad 0.798 \quad \mathrm{~g}, \quad 2.1 \quad \mathrm{mmol})$, tetrakis(triphenylphosphine)palladium ( $81 \mathrm{mg}, 0.07 \mathrm{mmol}, 10 \mathrm{~mol} \%$ ), and $\mathrm{Na}_{2} \mathrm{CO}_{3}(1.58 \mathrm{~g}, 15 \mathrm{mmol})$ were placed under argon. A mixture of toluene $(20 \mathrm{~mL})$, ethanol $(7 \mathrm{~mL})$, and water $(10 \mathrm{~mL})$ was added to the flask, and the solution was stirred at $110{ }^{\circ} \mathrm{C}$ for $16 \mathrm{~h}$. After quenching by addition of water $(50 \mathrm{~mL})$, the mixture was extracted with $\mathrm{CHCl}_{3}(30 \mathrm{~mL} \times 3)$. The combined organic extracts were washed with brine, dried over anhydrous $\mathrm{Na}_{2} \mathrm{SO}_{4}$, and evaporated to dryness under reduced pressure. The residual gray solid was subjected to column chromatography on $\mathrm{SiO}_{2}\left(\mathrm{CHCl}_{3}\right)$ followed by washing with $n$ hexane $(300 \mathrm{~mL})$. Recrystallization from $\mathrm{CHCl}_{3}-n$-hexane allowed DPA-C1-DPA as colorless powder $(0.484 \mathrm{~g}, 0.69 \mathrm{mmol})$ in $98 \%$ yield. mp $403{ }^{\circ} \mathrm{C} ;{ }^{1} \mathrm{H}$ NMR $\left(400 \mathrm{MHz}, \mathrm{CDCl}_{3}\right) \delta_{\text {ppm }} 2.00(\mathrm{~s}, 6 \mathrm{H}), 7.33(\mathrm{ddd}, J=7.1,6.2,1.8 \mathrm{~Hz}, 4 \mathrm{H}), 7.37$ (ddd, $J=$ 7.1, 6.2, $1.8 \mathrm{~Hz}, 4 \mathrm{H}), 7.49$ (AA'BB', $J=8.0 \mathrm{~Hz}, 4 \mathrm{H}), 7.50$ (m, 4H), $7.55(\mathrm{~m}, 2 \mathrm{H}), 7.60$ (m, 4H), 7.64 (AA'BB', $J=8.0 \mathrm{~Hz}$, $4 \mathrm{H}), 7.70(\mathrm{dd}, J=7.1,1.8 \mathrm{~Hz}, 4 \mathrm{H}), 7.80(\mathrm{dd}, J=7.1,1.8 \mathrm{~Hz}, 4 \mathrm{H}) ;{ }^{13} \mathrm{C}$ NMR $\left(100 \mathrm{MHz}, \mathrm{CDCl}_{3}\right) \delta_{\mathrm{ppm}} 31.2(2 \mathrm{C}), 43.2,124.94$ (4C), 124.99 (4C), 126.96 (8C), 127.1 (4C), 127.4 (2C), 128.4 (4C), 129.94 (4C), 130.02 (4C), 131.06 (4C), 131.35 (4C), 136.3 (2C), 137.0 (2C), 137.2 (2C), 139.2 (2C), 149.9 (2C); Anal. Calcd for $\mathrm{C}_{55} \mathrm{H}_{40}$ : C, 94.25; H, 5.75. Found: C, 94.07; H, 6.10, N, 0.16; HR-mass (ESI) $m / z[\mathrm{M}+\mathrm{H}]^{+}$Calcd for $\mathrm{C}_{55} \mathrm{H}_{40}$ : 701.3208; Found: 701.3286.

9,10-Bis(4-(10-phenylanthracene-9-yl)phenyl)tetracyclo[6.2.0.0 $\left.{ }^{4,10} \cdot 0^{5,9}\right]$ decane (DPA-C2-DPA): In a $100 \mathrm{~mL}$ threenecked flask, 9,10-bis(4-bromophenyl)tetracyclo[6.2.0.0 $\left.{ }^{4,10} .0^{5,9}\right]$ decane $(3,0.20 \mathrm{~g}, 0.45 \mathrm{mmol})$, (10-phenylanthracen-9yl)boronic acid (4, $0.28 \mathrm{~g}, 0.94 \mathrm{mmol})$, tetrakis(triphenylphosphine)palladium (58 $\mathrm{mg}, 0.05 \mathrm{mmol}, 5 \mathrm{~mol} \%)$, and $\mathrm{K}_{2} \mathrm{CO}_{3}(3.31$ $\mathrm{g}, 24 \mathrm{mmol})$ were placed under argon. A mixture of toluene $(8 \mathrm{~mL})$, ethanol $(8 \mathrm{~mL})$, and water $(2 \mathrm{~mL})$ was added to the flask, and the solution was stirred at $90{ }^{\circ} \mathrm{C}$ for $24 \mathrm{~h}$. After quenching by addition of water $(50 \mathrm{~mL})$, the mixture was extracted with $\mathrm{CHCl}_{3}(50 \mathrm{~mL} \times 1)$. The combined organic extracts were washed with brine, dried over anhydrous $\mathrm{Na}_{2} \mathrm{SO}_{4}$, and evaporated to dryness under reduced pressure. The residue was subjected to column chromatography on $\mathrm{SiO}_{2}\left(\mathrm{CHCl}_{3}\right)$. Recrystallization 
from benzene-ethanol allowed DPA-C2-DPA as pale yellow powder $(231 \mathrm{mg}, 0.29 \mathrm{mmol})$ in $65 \%$ yield. mp $>300{ }^{\circ} \mathrm{C} ;{ }^{1} \mathrm{H}$ NMR $\left(400 \mathrm{MHz}, \mathrm{CDCl}_{3}\right) \delta_{\mathrm{ppm}} 2.04$ (br d, $\left.J=8.4 \mathrm{~Hz}, 4 \mathrm{H}\right), 2.25$ (br d, $\left.J=8.4 \mathrm{~Hz} \mathrm{~Hz}, 4 \mathrm{H}\right), 3.41$ (br s, 4H), 7.17-7.20 (m, 4H), 7.21-7.26 (m, 4H), 7.39 (br s, 8H), 7.46 (AA'BB', $J=7.2 \mathrm{~Hz}, 4 \mathrm{H}), 7.51-7.62(\mathrm{~m}, 6 \mathrm{H}), 7.65(\mathrm{~m}, 4 \mathrm{H}), 7.67$ (m, 4H); ${ }^{13} \mathrm{C} \mathrm{NMR}$ $\left(100 \mathrm{MHz}, \mathrm{CDCl}_{3}\right) \delta_{\mathrm{ppm}} 27.3$ (4C), 44.5 (4C), 61.5 (2C), 124.89 (4C), 124.98 (4C), 126.7 (4C), 126.8 (4C), 127.0 (4C), 127.4 (2C), 128.4 (4C), 129.8 (4C), 130.0 (4C), 130.9 (4C), 131.33 (4C), 136.1 (2C), 136.8 (2C), 137.3 (2C), 139.2 (2C), 141.5 (2C); Anal. Calcd for $\mathrm{C}_{62} \mathrm{H}_{46}$ : C, 94.14; H, 5.86. Found: C, 93.40; H, 5.91; HR-mass (ESI) $\mathrm{m} / z[\mathrm{M}+\mathrm{H}]^{+}$Calcd for $\mathrm{C}_{62} \mathrm{H}_{46}$ : 791.3678; Found: 791.3683.

\section{Phosphorescence Quenching Experiments}

Phosphorescence quenching data was obtained based on analysis of phosphorescence decay curve of ${ }^{3} \mathrm{PtOEP} *$ upon laser flash photolysis (LFP). LFP system was comprised of a Spectra Physics Quanta-Ray GC-100 Nd:YAG pulsed laser (532 $\mathrm{nm}, 3 \mathrm{~mJ}$ pulse $^{-1}$, fwhm $=8 \mathrm{~ns}$ ) and UNISOKU TSP-1000 detection system with a suitable cutoff filter. The sample solutions were degassed with three freeze $(77 \mathrm{~K})$-pump $(0.1 \mathrm{mmHg})$-thaw (room temperature) cycles before experiments. Phosphorescence lifetimes of ${ }^{3} \mathrm{PtOEP} *$ in the absence $\left(\tau_{0}\right)$ and presence $(\tau)$ of a quencher $(\mathrm{Q})$ were obtained by using single exponential fitting. Stern-Volmer plot analysis (Fig. S1a) using eqn S1 affords $k_{\mathrm{ET}}$ for each Qs, e.g., DPA, DPA-C1-DPA, DPA-C2-DPA, and DPA-C3-DPA. $\Phi_{\mathrm{ET}}$ value calculated by eqn S2 was 0.978 when the concentrations of DPA, DPAC1-DPA, DPA-C2-DPA, and DPA-C3-DPA are 0.7, 0.4, 0.5, and $0.5 \mathrm{mM}$, respectively (Fig. S1b).

$$
\begin{aligned}
& \tau_{0} / \tau=\mathrm{a}+k_{\mathrm{ET}} \tau_{0}[\mathrm{Q}] \\
& \Phi_{\mathrm{ET}}=1-1 /\left(1+k_{\mathrm{q}} \tau_{0}[\mathrm{Q}]\right)
\end{aligned}
$$

(a)

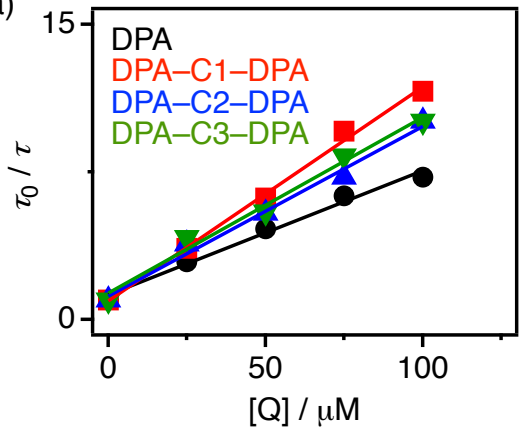

(b)

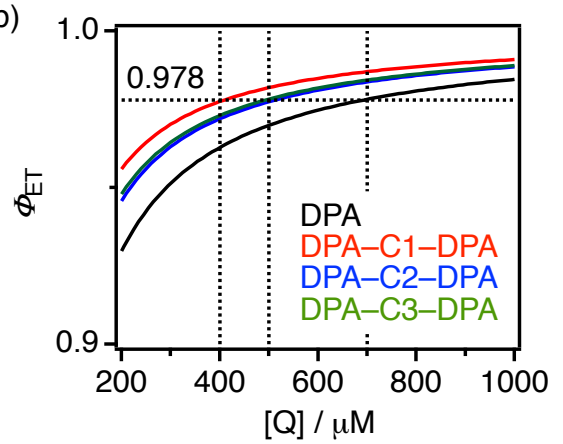

Fig. S1. (a) Stern-Volmer plots for phosphorescence quenching of ${ }^{3} \mathrm{PtOEP} *$ in the presence of Q, e.g., DPA (black), DPAC1-DPA (red), DPA-C2-DPA (blue), and DPA-C3-DPA (green). (b) Relationships of $\Phi_{\mathrm{ET}}$ against [Q] calculated by parameterized eqn $\mathrm{S} 2$.

Table S1. Fitting Parameters of eqn S1 in Stern-Volmer Plots for Phosphorescence Quenching of ${ }^{3} \mathrm{PtOEP} *$ by four Qs

\begin{tabular}{cccc}
\hline $\mathrm{Q}$ & $\mathrm{a}$ & $k_{\mathrm{ET}} / 10^{9} \mathrm{M}^{-1} \mathrm{~s}^{-1}$ & $R^{2}$ \\
\hline DPA & 1.24 & 1.65 & 0.987 \\
DPA-C1-DPA & 0.96 & 2.84 & 0.996 \\
DPA-C2-DPA & 1.20 & 2.25 & 0.989 \\
DPA-C3-DPA & 1.30 & 2.33 & 0.986 \\
\hline
\end{tabular}




\section{Raw Data of UC Luminescence Spectra}

UC luminescence observation was made using Ocean Optics USB4000 multichannel detector upon CW-laser (RGB Photonics, Minilas Fiber $520 \mathrm{~nm}$ ) excitation. The sample solutions were degassed with three freeze (77 K)-pump (0.1 $\mathrm{mmHg}$ )-thaw (room temperature) cycles before experiments. Laser intensity was corrected using power meter.
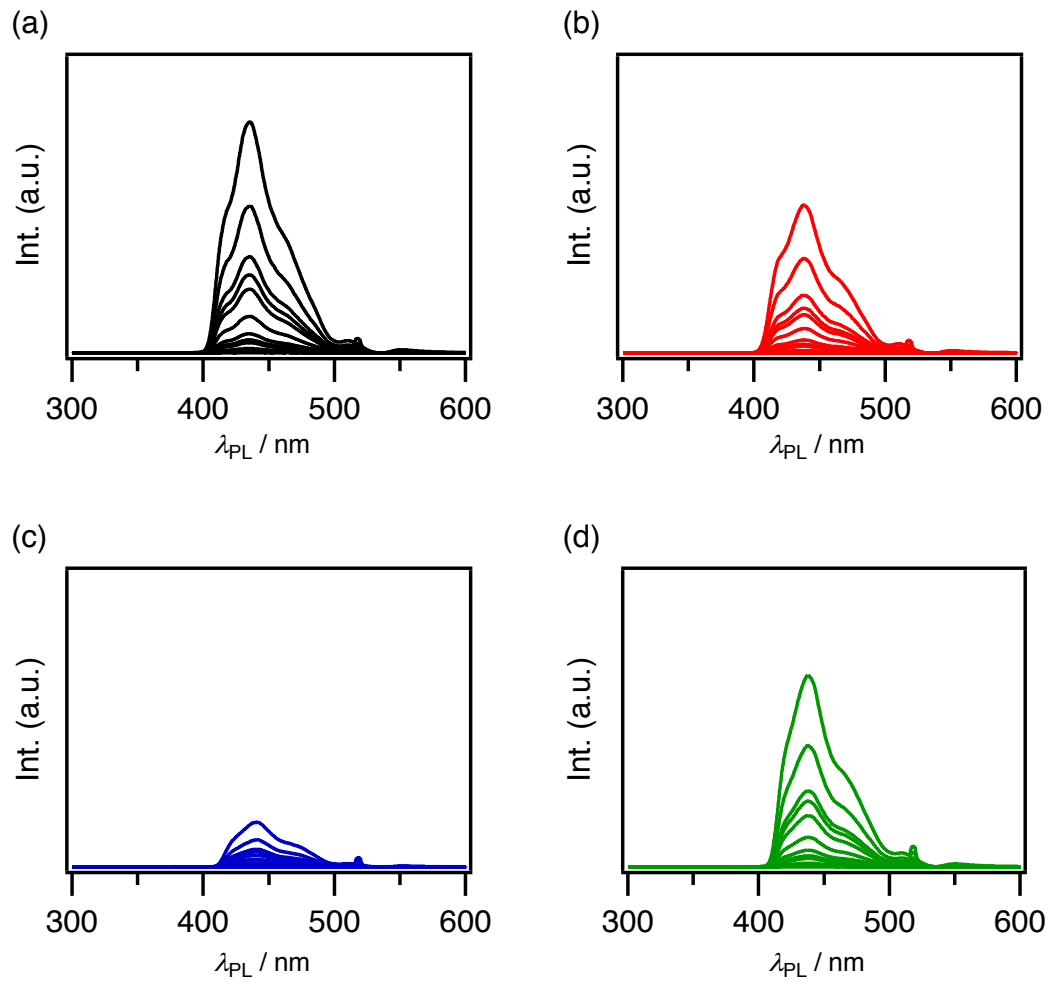

Fig. S2. UC luminescence spectra upon 520-nm excitation of a degassed $\mathrm{CH}_{2} \mathrm{Cl}_{2}$ solution of DPA (a, $0.7 \mathrm{mM}$ ) or DPA-C1DPA (b, 0.4 mM), DPA-C2-DPA (c, 0.5 mM), or DPA-C3-DPA (d, 0.5 mM) containing PtOEP (0.05 mM).

\section{Determination of Triplet Lifetimes under the Benzophenone-sensitization Conditions}

(a)

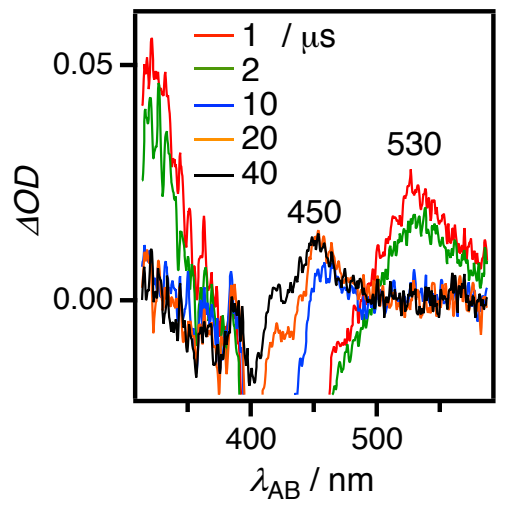

(b)

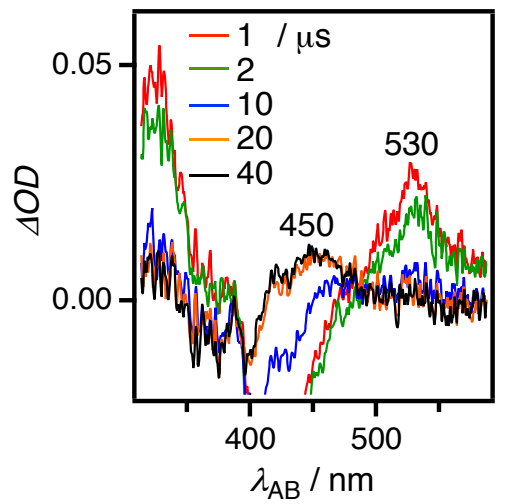

Fig. S3. Transient absorption spectra of a benzene solution of DPA (a, $50 \mu \mathrm{M})$ and DPA-C1-DPA (b, $25 \mu \mathrm{M})$ containing benzophenone $(1.0 \mathrm{mM})$ upon LFP $(355 \mathrm{~nm})$. The 450 - and 530-nm absorption bands are assigned to the ${ }^{3} \mathrm{DPA} *$ moiety and triplet excited benzophenone, respectively. 


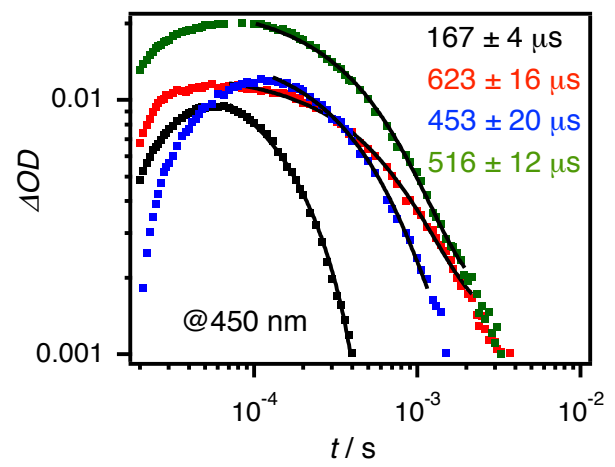

Fig. S4. Decay profiles of the absorption of the ${ }^{3} \mathrm{DPA}^{*}$ moiety at $450 \mathrm{~nm}$ under the benzophenone-sensitized conditions for DPA (black), DPA-C1-DPA (red), DPA-C2-DPA (blue), and DPA-C3-DPA (green). Longer triplet lifetimes of DPA-CnDPA are observed, as compared with those observed under the PtOEP-sensitization conditions.

\section{NMR Spectra}

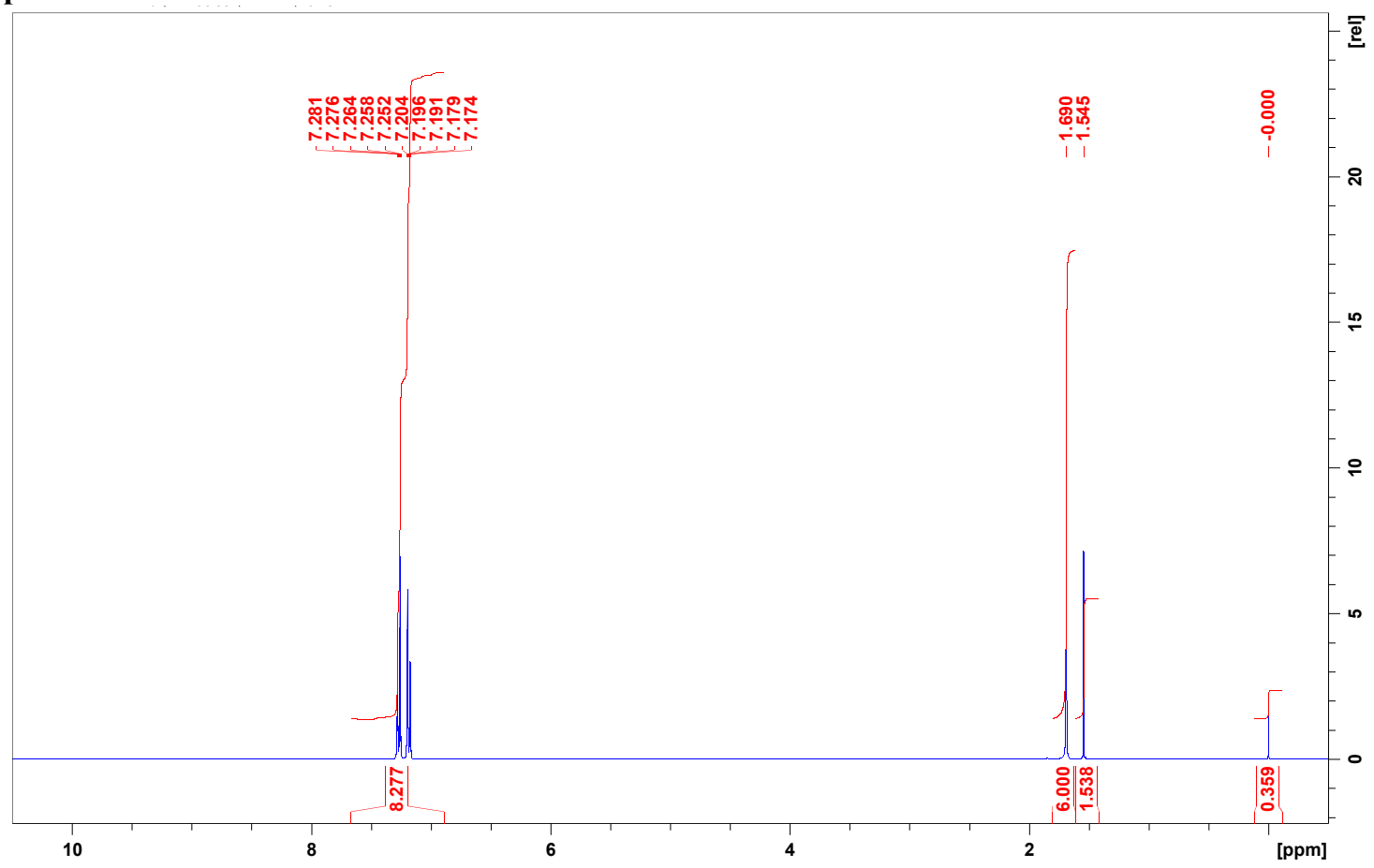

Fig. S5. ${ }^{1} \mathrm{H}$ NMR spectrum of $1\left(400 \mathrm{MHz}, \mathrm{CDCl}_{3}\right)$. 


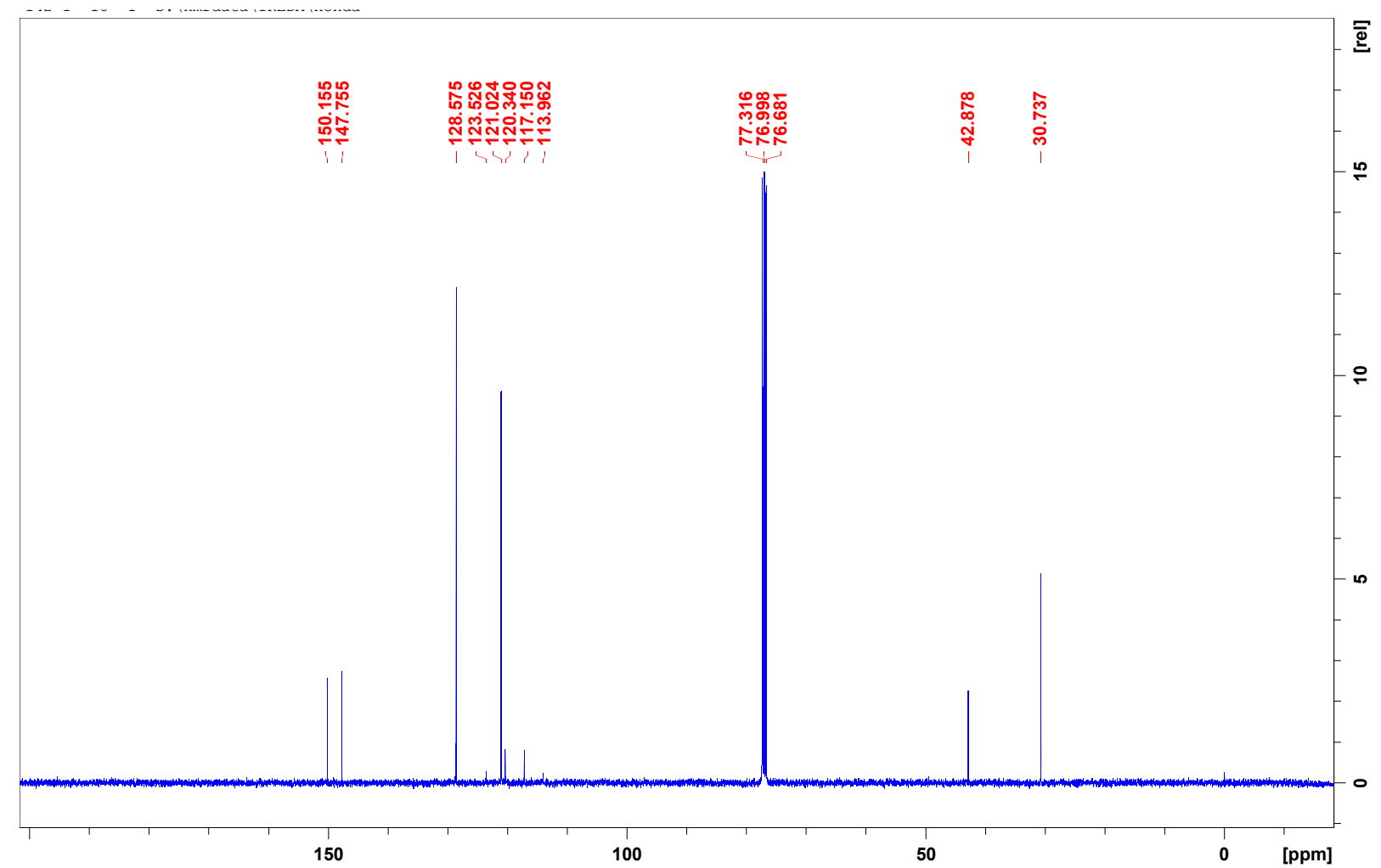

Fig. S6. ${ }^{13} \mathrm{C}$ NMR spectrum of $1\left(100 \mathrm{MHz}, \mathrm{CDCl}_{3}\right)$

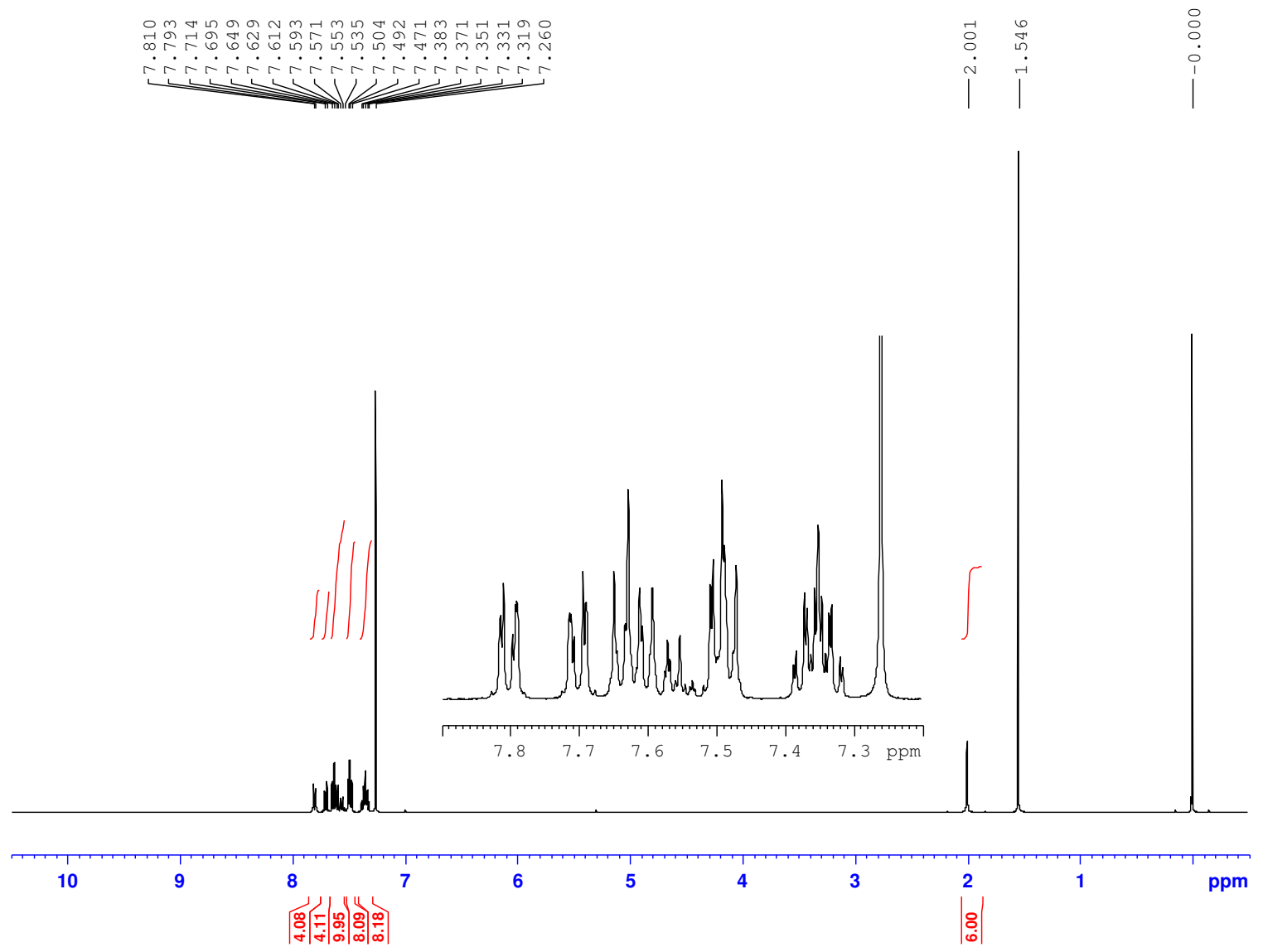

Fig. S7. ${ }^{1} \mathrm{H}$ NMR spectrum of DPA-C1-DPA (400 MHz, $\left.\mathrm{CDCl}_{3}\right)$. 


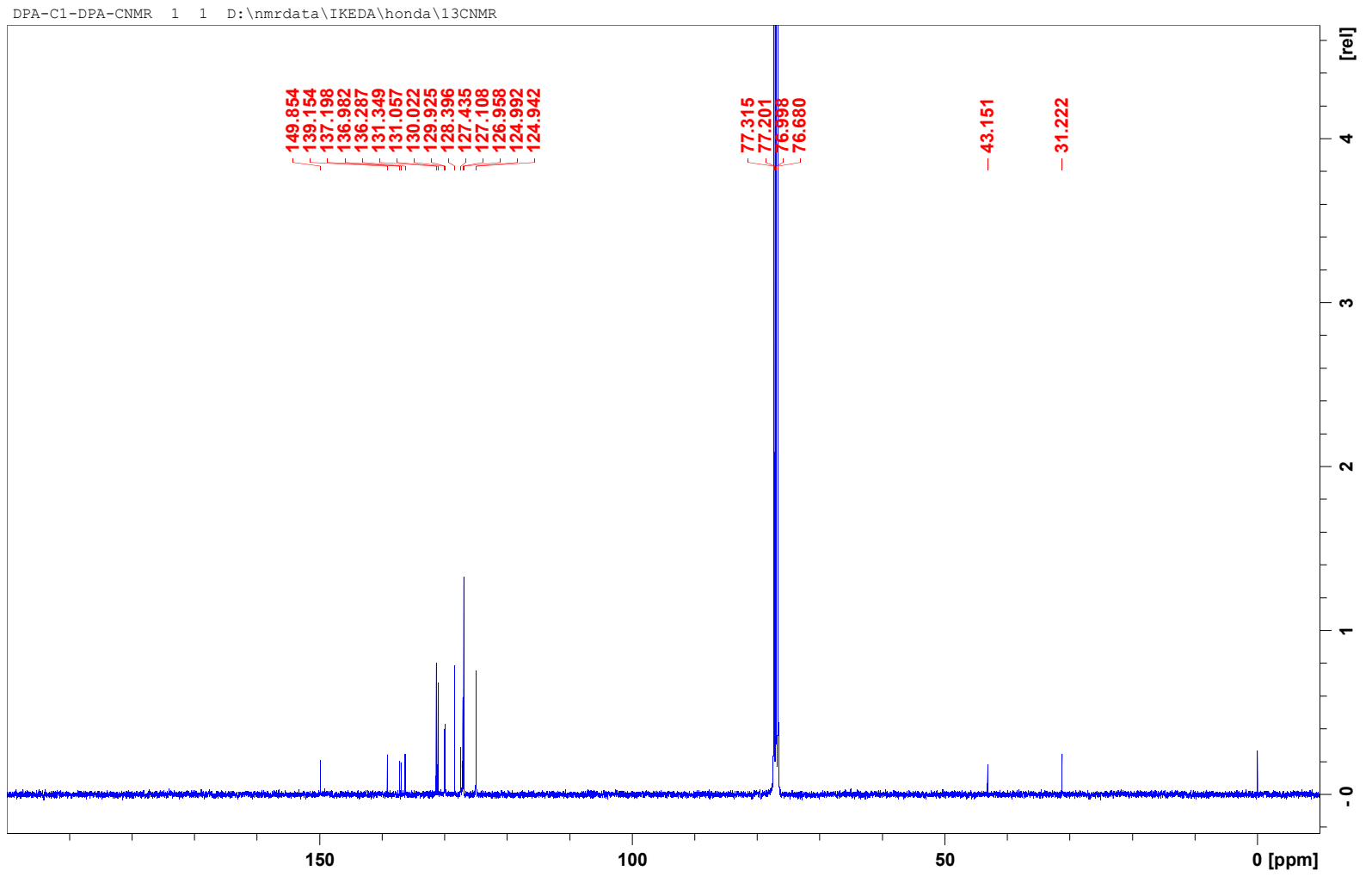

Fig. S8. ${ }^{13} \mathrm{C}$ NMR spectrum of DPA-C1-DPA $\left(100 \mathrm{MHz}, \mathrm{CDCl}_{3}\right)$.

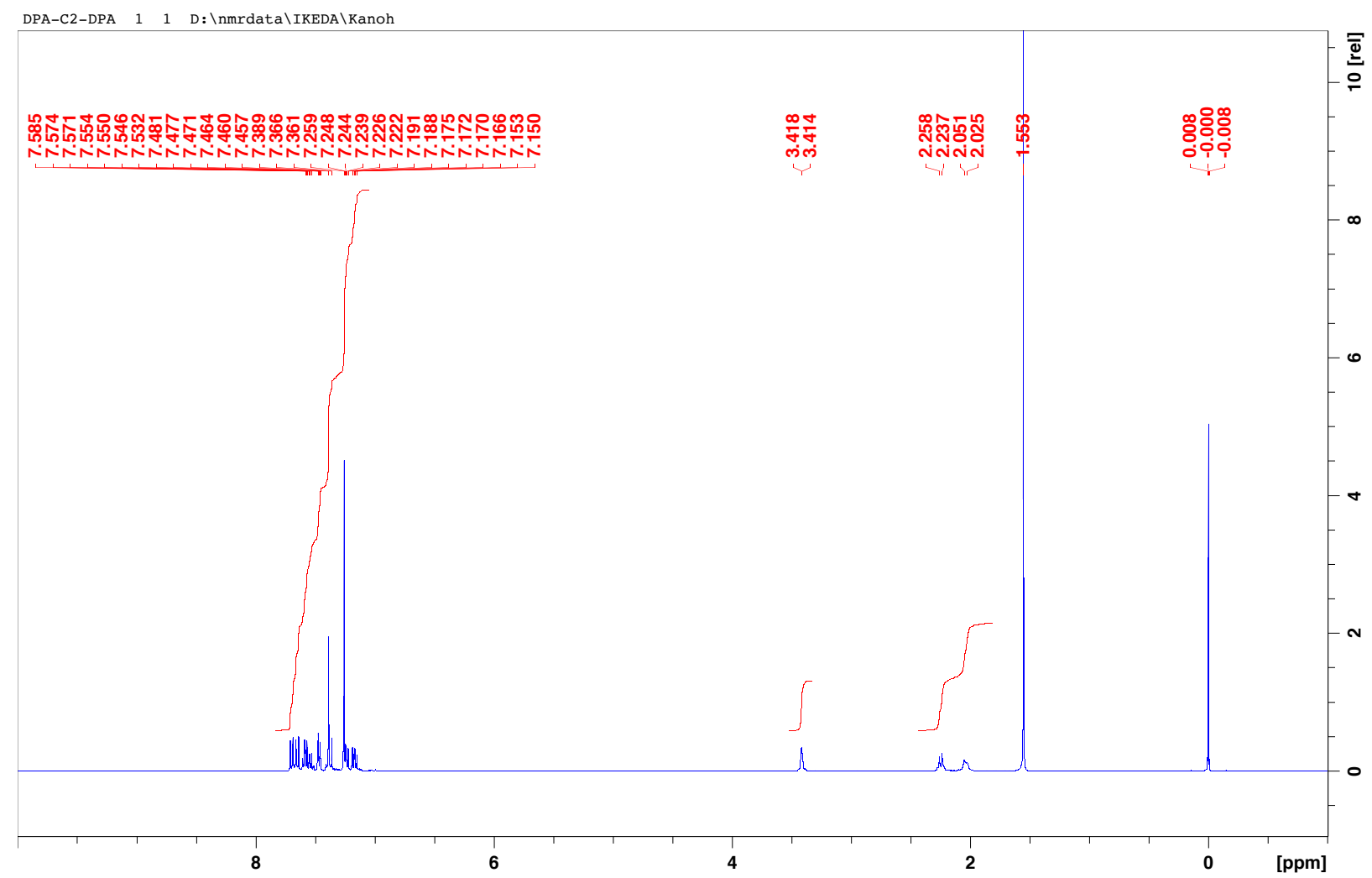

Fig. S9. ${ }^{1} \mathrm{H}$ NMR spectrum of DPA-C2-DPA (400 MHz, $\left.\mathrm{CDCl}_{3}\right)$. 


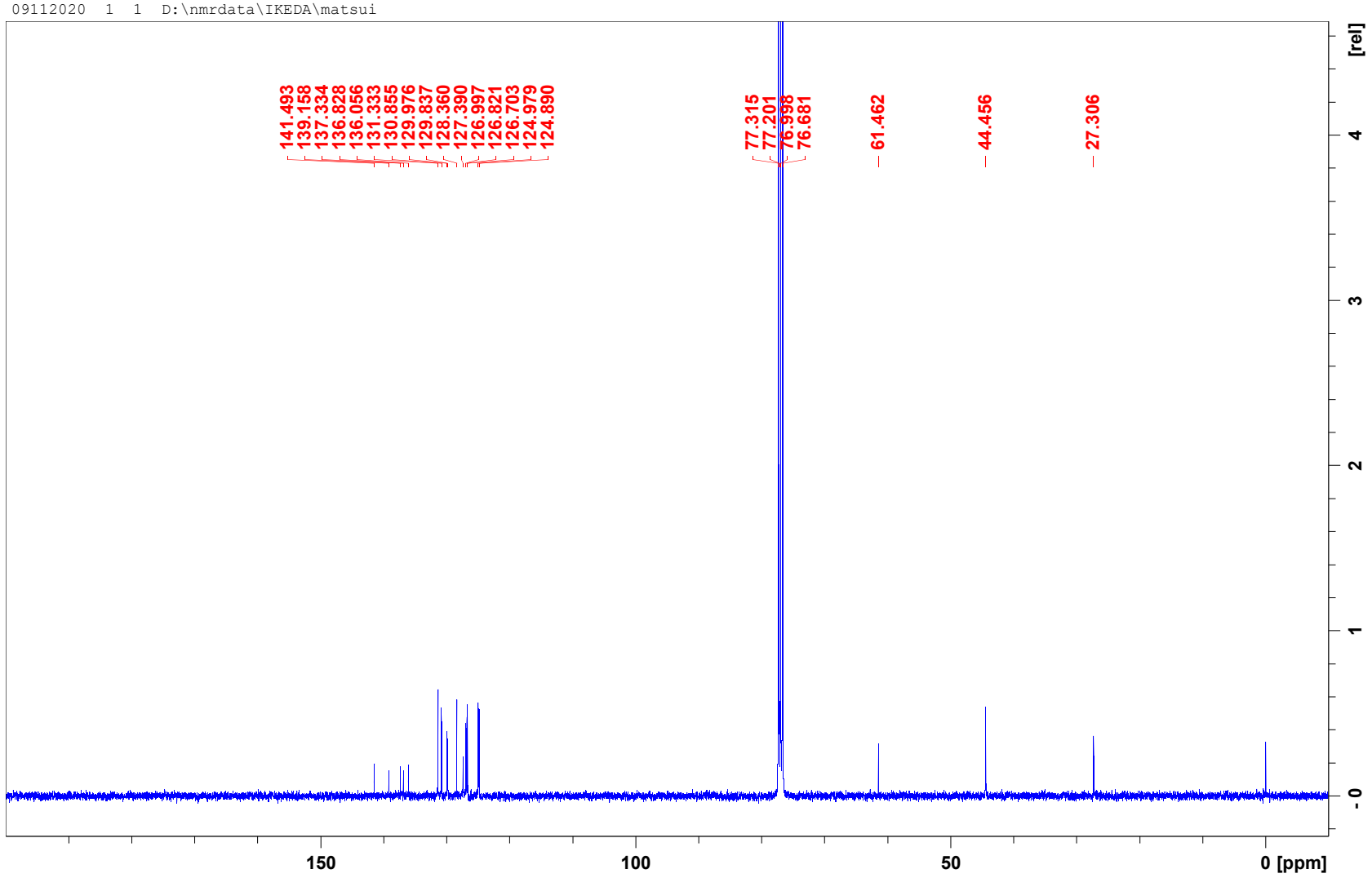

Fig. S10. ${ }^{13} \mathrm{C}$ NMR spectrum of DPA-C2-DPA $\left(100 \mathrm{MHz}, \mathrm{CDCl}_{3}\right)$. 


\section{Theoretical Calculations}

DFT calculations are carried out using a Gaussian 09W program with a B3LYP functional and 6-311G* basis set. Successful geometry optimization was achieved to afford no imaginary frequency.

$L+1$
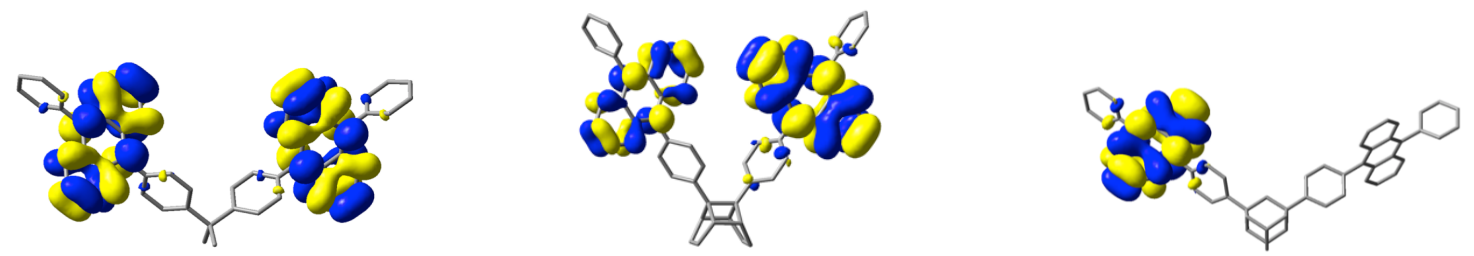

$\mathbf{L}$

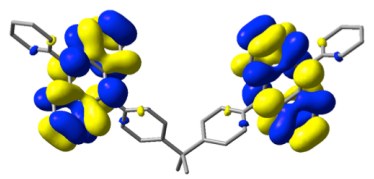

H
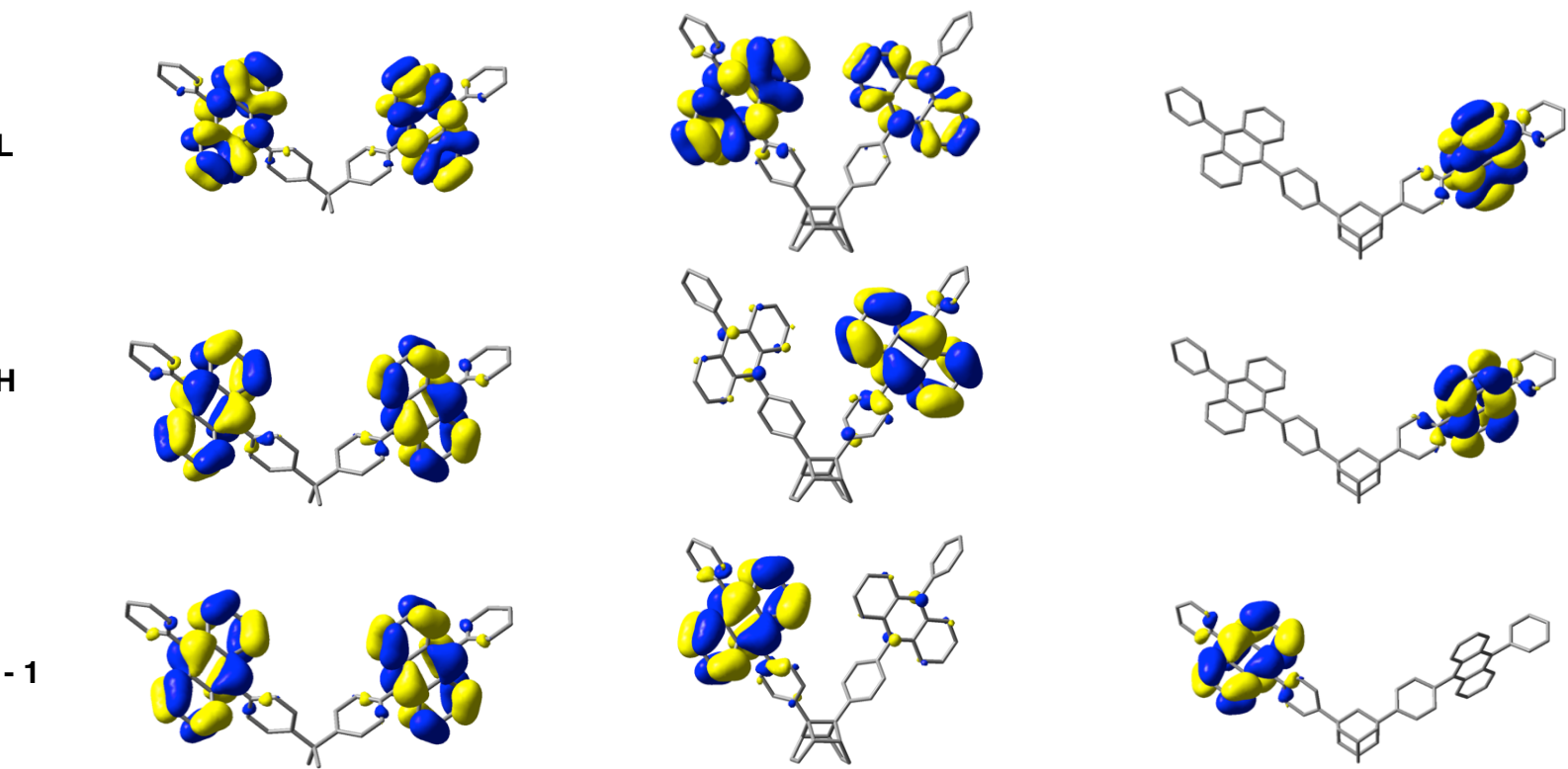

Fig. S11. Frontier molecular orbital coefficient distributions of DPA-C1-DPA, DPA-C2-DPA, and DPA-C3-DPA(B3LYP/6$\left.311 \mathrm{G}^{*}\right)$. $\mathrm{H}$ and $\mathrm{L}$ refer to the HOMO and the LUMO.

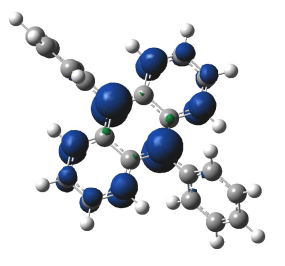

${ }^{3} \mathrm{DPA}^{*}$

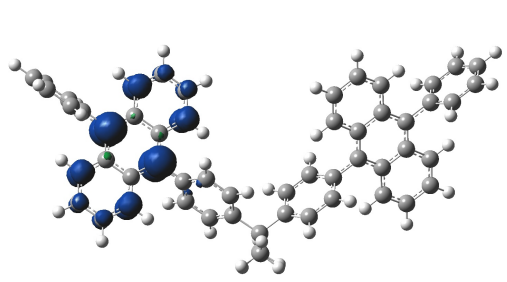

${ }^{3} \mathrm{DPA}^{*}-\mathrm{C} 1-\mathrm{DPA}$

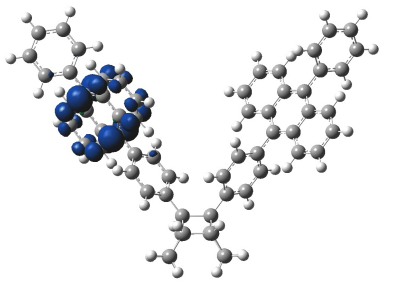

${ }^{3} \mathrm{DPA}^{*}-\mathrm{C} 2-\mathrm{DPA}$

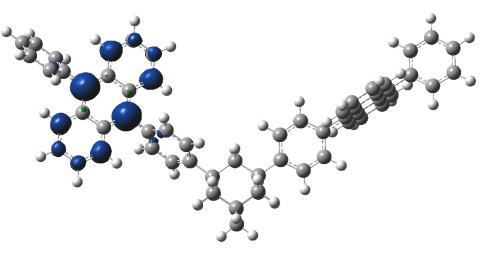

${ }^{3} \mathrm{DPA}^{*}-\mathrm{C} 3-\mathrm{DPA}$

Fig. S12. Spin distributions of ${ }^{3} \mathrm{DPA}^{*},{ }^{3} \mathrm{DPA}{ }^{*}-\mathrm{C} 1-\mathrm{DPA},{ }^{3} \mathrm{DPA}{ }^{*}-\mathrm{C} 2-\mathrm{DPA}$, and ${ }^{3} \mathrm{DPA}{ }^{*}-\mathrm{C} 3-\mathrm{DPA}\left(\mathrm{UB} 3 \mathrm{LYP} / 6-311 \mathrm{G}^{*}\right)$. The spin densities are localized to the single DPA moiety. 


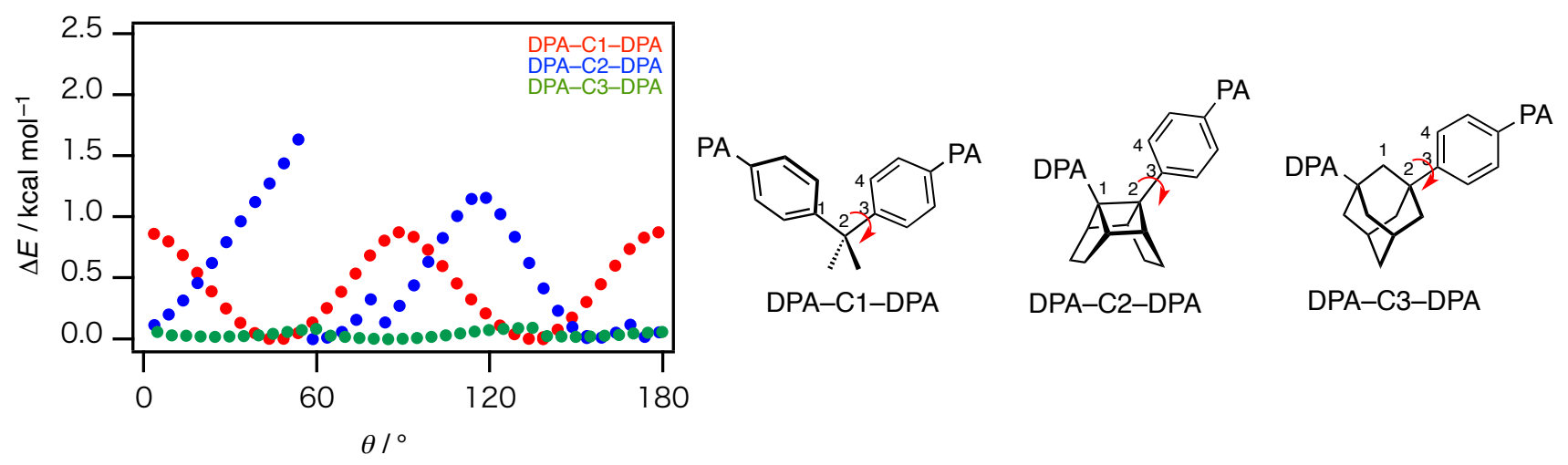

Fig. S13. Energy profiles against dihedral angle $\theta=\angle \mathrm{C} 1-\mathrm{C} 2-\mathrm{C} 3-\mathrm{C} 4$, calculated by using PM5 level of theory. PA refers to the 10-phenylanthracen-9-yl group. 
Table S2. The Cartesian Coordinates (in $\AA$ ) of DPA-C1DPA Optimized by B3LYP/6-311G* (The Sum of Electronic and Zero-point Energies is -2119.223105 Hartree.)

\begin{tabular}{|c|c|c|c|}
\hline \multirow{2}{*}{ Atom } & \multicolumn{3}{|c|}{ Coordinates } \\
\hline & $\mathrm{X}$ & $\mathrm{Y}$ & Z \\
\hline $\mathrm{C}$ & -0.14776 & 4.83766 & -1.23831 \\
\hline $\mathrm{C}$ & 0.00003 & 3.92156 & 0.00025 \\
\hline $\mathrm{H}$ & 0.68729 & 5.54322 & -1.31485 \\
\hline $\mathrm{H}$ & -0.20117 & 4.26402 & -2.16901 \\
\hline $\mathrm{H}$ & -1.07076 & 5.42046 & -1.15372 \\
\hline $\mathrm{C}$ & 1.25729 & 3.03168 & -0.07389 \\
\hline $\mathrm{C}$ & 0.14788 & 4.83737 & 1.23901 \\
\hline $\mathrm{C}$ & -1.25729 & 3.03174 & 0.07420 \\
\hline $\mathrm{H}$ & 1.07092 & 5.42014 & 1.15454 \\
\hline $\mathrm{H}$ & -0.68712 & 5.54296 & 1.31574 \\
\hline $\mathrm{H}$ & 0.20129 & 4.26351 & 2.16958 \\
\hline $\mathrm{C}$ & 2.25189 & 3.18849 & -1.04616 \\
\hline $\mathrm{C}$ & 1.45468 & 2.03435 & 0.89587 \\
\hline $\mathrm{C}$ & 2.59304 & 1.23374 & 0.89600 \\
\hline $\mathrm{C}$ & 3.39506 & 2.38398 & -1.05128 \\
\hline $\mathrm{C}$ & 3.58773 & 1.39362 & -0.08183 \\
\hline $\mathrm{H}$ & 0.69675 & 1.87302 & 1.65818 \\
\hline $\mathrm{H}$ & 2.71489 & 0.46985 & 1.65955 \\
\hline $\mathrm{H}$ & 2.14896 & 3.94091 & -1.82106 \\
\hline $\mathrm{H}$ & 4.14676 & 2.52698 & -1.82325 \\
\hline $\mathrm{C}$ & 4.80863 & 0.52692 & -0.09198 \\
\hline $\mathrm{C}$ & -1.45481 & 2.03471 & -0.89585 \\
\hline $\mathrm{C}$ & -2.25179 & 3.18828 & 1.04662 \\
\hline $\mathrm{C}$ & -3.39497 & 2.38379 & 1.05163 \\
\hline $\mathrm{C}$ & -2.59320 & 1.23413 & -0.89609 \\
\hline $\mathrm{C}$ & -3.58777 & 1.39373 & 0.08190 \\
\hline $\mathrm{H}$ & -2.14876 & 3.94047 & 1.82174 \\
\hline $\mathrm{H}$ & -4.14659 & 2.52657 & 1.82373 \\
\hline $\mathrm{H}$ & -0.69696 & 1.87358 & -1.65828 \\
\hline $\mathrm{H}$ & -2.71514 & 0.47047 & -1.65985 \\
\hline $\mathrm{C}$ & -4.80866 & 0.52702 & 0.09197 \\
\hline $\mathrm{C}$ & 4.74856 & -0.76918 & -0.65015 \\
\hline $\mathrm{C}$ & 6.02008 & 1.00462 & 0.45455 \\
\hline $\mathrm{C}$ & 5.92692 & -1.60858 & -0.65876 \\
\hline $\mathrm{C}$ & 3.54254 & -1.28804 & -1.22471 \\
\hline $\mathrm{C}$ & 7.20120 & 0.16920 & 0.43585 \\
\hline $\mathrm{C}$ & 7.13959 & -1.12796 & -0.11841 \\
\hline $\mathrm{C}$ & 5.82056 & -2.92217 & -1.22174 \\
\hline $\mathrm{C}$ & 3.48914 & -2.54828 & -1.75765 \\
\hline $\mathrm{C}$ & 4.64369 & -3.37899 & -1.75218 \\
\hline $\mathrm{H}$ & 2.65983 & -0.65840 & -1.23178 \\
\hline $\mathrm{C}$ & 8.41458 & 0.69668 & 0.98653 \\
\hline $\mathrm{C}$ & 8.36157 & -1.99486 & -0.13158 \\
\hline $\mathrm{H}$ & 6.69860 & -3.55848 & -1.22062 \\
\hline $\mathrm{H}$ & 2.56169 & -2.91781 & -2.18705 \\
\hline $\mathrm{H}$ & 4.58926 & -4.37975 & -2.17245 \\
\hline $\mathrm{C}$ & 8.46537 & 1.95153 & 1.53217 \\
\hline $\mathrm{H}$ & 9.30474 & 0.07784 & 0.96480 \\
\hline $\mathrm{C}$ & 7.30015 & 2.76659 & 1.56572 \\
\hline $\mathrm{H}$ & 9.39823 & 2.32804 & 1.94340 \\
\hline $\mathrm{C}$ & 6.12035 & 2.30694 & 1.04424 \\
\hline $\mathrm{H}$ & 7.34909 & 3.75825 & 2.00775 \\
\hline $\mathrm{H}$ & 5.23458 & 2.93179 & 1.07236 \\
\hline $\mathrm{C}$ & 9.18269 & -2.05950 & -1.26751 \\
\hline $\mathrm{C}$ & 8.71031 & -2.75886 & 0.99250 \\
\hline $\mathrm{C}$ & 9.84915 & -3.56587 & 0.98158 \\
\hline $\mathrm{C}$ & 10.32220 & -2.86559 & -1.27918 \\
\hline $\mathrm{C}$ & 10.65856 & -3.62115 & -0.15449 \\
\hline $\mathrm{H}$ & 8.08138 & -2.71696 & 1.87783 \\
\hline $\mathrm{H}$ & 10.10308 & -4.15167 & 1.86126 \\
\hline $\mathrm{H}$ & 8.92300 & -1.47176 & -2.14401 \\
\hline $\mathrm{H}$ & 10.94729 & -2.90233 & -2.16767 \\
\hline $\mathrm{H}$ & 11.54560 & -4.24900 & -0.16338 \\
\hline
\end{tabular}

$\begin{array}{llll}\text { C } & -4.74868 & -0.76895 & 0.65044\end{array}$

$\begin{array}{llll}\text { C } & -7.20111 & 0.16914 & -0.43630\end{array}$

$\begin{array}{llll}\mathrm{C} & -6.12017 & 2.30679 & -1.04486\end{array}$

$\begin{array}{llll}\mathrm{C} & -5.92703 & -1.60837 & 0.65901\end{array}$

$\begin{array}{llll}\text { C } & -7.13960 & -1.12789 & 0.11828\end{array}$

$\begin{array}{llll}\text { C } & -8.41438 & 0.69648 & -0.98735\end{array}$

$\begin{array}{llll}\mathrm{C} & -7.29987 & 2.76632 & -1.56667\end{array}$

$\begin{array}{llll}\mathrm{C} & -8.46507 & 1.95122 & -1.53325\end{array}$

$\begin{array}{llll}\mathrm{H} & -5.23442 & 2.93167 & -1.07286\end{array}$

$\begin{array}{llll}\mathrm{C} & -5.82076 & -2.92183 & 1.22230\end{array}$

$\begin{array}{llll}\mathrm{C} & -8.36157 & -1.99480 & 0.13140\end{array}$

$\begin{array}{llll}\mathrm{H} & -9.30453 & 0.07763 & -0.96569\end{array}$

$\begin{array}{llll}\mathrm{H} & -7.34876 & 3.75790 & -2.00888\end{array}$

$\begin{array}{llll}\mathrm{H} & -9.39786 & 2.32763 & -1.94476\end{array}$

$\begin{array}{llll}\mathrm{C} & -4.64398 & -3.37851 & 1.75305\end{array}$

$\begin{array}{llll}\mathrm{H} & -6.69879 & -3.55815 & 1.22117\end{array}$

$\begin{array}{llll}\mathrm{C} & -3.48944 & -2.54779 & 1.75853\end{array}$

$\begin{array}{llll}\mathrm{H} & -4.58961 & -4.37918 & 2.17356\end{array}$

$\begin{array}{llll}\mathrm{H} & -2.56205 & -2.91723 & 2.18815\end{array}$

$\begin{array}{llll}\mathrm{C} & -3.54275 & -1.28767 & 1.22531\end{array}$

$\begin{array}{llll}\mathrm{H} & -2.66004 & -0.65802 & 1.23236\end{array}$

$\begin{array}{llll}\mathrm{C} & -2.66004 & -0.65802 & 1.23236 \\ \mathrm{C} & -8.71012 & -2.75900 & -0.99260\end{array}$

$\begin{array}{llll}\text { C } & -9.18286 & -2.05926 & 1.26721\end{array}$

$\begin{array}{llll}\text { C } & -10.32237 & -2.86536 & 1.27885\end{array}$

$\begin{array}{llll}\text { C } & -9.84896 & -3.56602 & -0.98172\end{array}$

$\begin{array}{llll}\text { C } & -10.65854 & -3.62111 & 0.15423\end{array}$

$\begin{array}{llll}\mathrm{H} & -8.92332 & -1.47136 & 2.14365\end{array}$

$\begin{array}{llll}\mathrm{H} & -10.94759 & -2.90196 & 2.16725\end{array}$

$\mathrm{H} \quad-8.08106 \quad-2.71724 \quad-1.87784$

$\begin{array}{llll}\mathrm{H} & -10.10274 & -4.15198 & -1.86135\end{array}$

$\begin{array}{llll}H & -11.54558 & -4.24897 & 0.16309\end{array}$

$\begin{array}{llll}\mathrm{C} & -0.14776 & 4.83766 & -1.23831\end{array}$

$\begin{array}{llll}\mathrm{C} & 0.00003 & 3.92156 & 0.00025\end{array}$

$\begin{array}{llll}\mathrm{H} & 0.68729 & 5.54322 & -1.31485\end{array}$

$\begin{array}{llll}\mathrm{H} & -0.20117 & 4.26402 & -2.16901\end{array}$

$\begin{array}{llll}\mathrm{H} & -1.07076 & 5.42046 & -1.15372\end{array}$

$\begin{array}{llll}\mathrm{C} & 1.25729 & 3.03168 & -0.07389\end{array}$

$\begin{array}{llll}\mathrm{C} & 0.14788 & 4.83737 & 1.23901\end{array}$

$\begin{array}{llll}\text { C } & -1.25729 & 3.03174 & 0.07420\end{array}$

$\mathrm{H} \quad 1.07092 \quad 5.42014 \quad 1.15454$

$\begin{array}{llll}\mathrm{H} & -0.68712 & 5.54296 & 1.31574\end{array}$

$\begin{array}{llll}\mathrm{H} & 0.20129 & 4.26351 & 2.16958\end{array}$

$\begin{array}{llll}\mathrm{C} & 2.25189 & 3.18849 & -1.04616\end{array}$

$\begin{array}{llll}\mathrm{C} & 1.45468 & 2.03435 & 0.89587\end{array}$

$\begin{array}{llll}\mathrm{C} & 2.59304 & 1.23374 & 0.89600\end{array}$

\begin{tabular}{llll}
$\mathrm{C}$ & 3.39506 & 2.38398 & -1.05128 \\
\hline
\end{tabular}

Table S3. The Cartesian Coordinates (in $\AA$ ) of DPA-C2DPA Optimized by B3LYP/6-311G* (The Sum of Electronic and Zero-point Energies is -2389.420140 Hartree.)

\begin{tabular}{cccc}
\hline & \multicolumn{3}{c}{ Coordinates } \\
Atom & $\mathrm{X}$ & $\mathrm{Y}$ & $\mathrm{Z}$ \\
\hline $\mathrm{C}$ & -0.73614 & 5.72783 & 1.20827 \\
$\mathrm{C}$ & -0.83201 & 4.72395 & 0.02287 \\
$\mathrm{H}$ & -1.05824 & 5.34211 & 2.17938 \\
$\mathrm{C}$ & -1.49711 & 7.00741 & 0.81168 \\
$\mathrm{C}$ & -1.64337 & 6.96037 & -0.73962 \\
$\mathrm{H}$ & -0.98254 & 7.91201 & 1.15439 \\
$\mathrm{H}$ & -2.48446 & 7.01834 & 1.28753 \\
$\mathrm{H}$ & -2.70069 & 6.87900 & -1.01735 \\
$\mathrm{C}$ & -0.90015 & 5.69679 & -1.19920 \\
$\mathrm{H}$ & -1.26627 & 7.86676 & -1.22637 \\
$\mathrm{H}$ & -1.34932 & 5.25371 & -2.09271 \\
$\mathrm{C}$ & -1.70210 & 3.50651 & 0.05641 \\
$\mathrm{C}$ & 0.76164 & 4.71968 & -0.11058 \\
$\mathrm{C}$ & 1.63211 & 3.50311 & -0.13091 \\
$\mathrm{C}$ & 0.67178 & 5.71028 & -1.30706
\end{tabular}




$\begin{array}{lccccccc}\mathrm{C} & 1.43701 & 6.99185 & -0.92495 & \mathrm{C} & -7.68709 & -5.53442 & -0.98132 \\ \mathrm{H} & 0.99409 & 5.30892 & -2.27206 & \mathrm{C} & -8.14236 & -6.04041 & 0.23756 \\ \mathrm{C} & 1.58504 & 6.96041 & 0.62680 & \mathrm{H} & -6.79076 & -3.70629 & 2.31482 \\ \mathrm{H} & 0.92418 & 7.89419 & -1.27605 & \mathrm{H} & -8.16878 & -5.76580 & 2.37701 \\ \mathrm{H} & 2.42378 & 6.99617 & -1.40219 & \mathrm{H} & -6.55862 & -3.98172 & -1.96338 \\ \mathrm{H} & 2.64231 & 6.87621 & 0.90390 & \mathrm{H} & -7.93423 & -6.04321 & -1.90952 \\ \mathrm{C} & 0.83551 & 5.70605 & 1.10074 & \mathrm{H} & -8.74581 & -6.94392 & 0.26307 \\ \mathrm{H} & 1.21362 & 7.87407 & 1.10428 & \mathrm{C} & 2.56231 & -3.76706 & -1.39783 \\ \mathrm{H} & 1.28230 & 5.26840 & 1.99822 & \mathrm{H} & 4.47718 & -4.59036 & -0.95911 \\ \mathrm{C} & -2.59385 & 3.24193 & 1.10518 & \mathrm{C} & 1.75111 & -2.59860 & -1.41872 \\ \mathrm{C} & -1.64053 & 2.57117 & -0.99189 & \mathrm{H} & 2.14821 & -4.71607 & -1.72806 \\ \mathrm{C} & -2.43077 & 1.42561 & -0.98916 & \mathrm{C} & 2.26063 & -1.39695 & -1.00224 \\ \mathrm{C} & -3.38673 & 2.09335 & 1.11091 & \mathrm{H} & 0.72317 & -2.66086 & -1.76517 \\ \mathrm{C} & -3.31794 & 1.16069 & 0.06693 & \mathrm{H} & 1.63890 & -0.50903 & -1.02580 \\ \mathrm{H} & -0.95628 & 2.73942 & -1.81972 & \mathrm{C} & 6.74755 & -4.41252 & 1.05271 \\ \mathrm{H} & -2.36008 & 0.72022 & -1.81300 & \mathrm{C} & 7.35212 & -3.98778 & -1.24376 \\ \mathrm{H} & -2.67687 & 3.93927 & 1.93388 & \mathrm{C} & 8.15206 & -5.13173 & -1.24542 \\ \mathrm{H} & -4.06505 & 1.91332 & 1.94087 & \mathrm{C} & 7.54659 & -5.55707 & 1.05192 \\ \mathrm{C} & -4.15151 & -0.08091 & 0.08411 & \mathrm{C} & 8.25127 & -5.91974 & -0.09735 \\ \mathrm{C} & 2.61676 & 3.29957 & -1.10771 & \mathrm{H} & 7.27544 & -3.37593 & -2.13868 \\ \mathrm{C} & 1.49172 & 2.51931 & 0.86379 & \mathrm{H} & 8.69751 & -5.40667 & -2.14452 \\ \mathrm{C} & 2.29820 & 1.38463 & 0.87878 & \mathrm{H} & 6.19994 & -4.13180 & 1.94850 \\ \mathrm{C} & 3.42832 & 2.16409 & -1.09334 & \mathrm{H} & 7.61803 & -6.16515 & 1.95005 \\ \mathrm{C} & 3.28194 & 1.18403 & -0.10265 & \mathrm{H} & 8.87369 & -6.81066 & -0.09827 \\ \mathrm{H} & 0.73277 & 2.63988 & 1.63257 & \mathrm{C} & -0.73614 & 5.72783 & 1.20827 \\ \mathrm{H} & 2.16538 & 0.63898 & 1.65838 & \mathrm{C} & -0.83201 & 4.72395 & 0.02287 \\ \mathrm{H} & 2.75976 & 4.03702 & -1.89270 & & & & \end{array}$

Table S4. The Cartesian Coordinates (in Å) of DPA-C3DPA Optimized by B3LYP/6-311G* (The Sum of Electronic and Zero-point Energies is -2390.667337 Hartree.)

\begin{tabular}{cccc}
\hline Atom & \multicolumn{3}{c}{ Coordinates } \\
& $\mathrm{X}$ & $\mathrm{Y}$ & $\mathrm{Z}$ \\
\hline $\mathrm{C}$ & 1.35099 & 4.20562 & 1.06616 \\
$\mathrm{H}$ & 2.23796 & 4.85291 & 1.04013 \\
$\mathrm{C}$ & 0.06757 & 5.05412 & 1.11239 \\
$\mathrm{C}$ & 1.35074 & 3.28240 & -0.18515 \\
$\mathrm{C}$ & -0.01152 & 5.93993 & -0.14457 \\
$\mathrm{C}$ & -1.16336 & 4.12889 & 1.17054 \\
$\mathrm{C}$ & 1.24989 & 4.18560 & -1.43874 \\
$\mathrm{C}$ & 0.07790 & 2.38733 & -0.13939 \\
$\mathrm{C}$ & -0.03073 & 5.04001 & -1.39415 \\
$\mathrm{C}$ & -1.26656 & 4.12265 & -1.35108 \\
$\mathrm{C}$ & -1.23000 & 3.21641 & -0.08845 \\
$\mathrm{H}$ & 1.42867 & 3.60200 & 1.97906 \\
$\mathrm{C}$ & 2.60673 & 2.40074 & -0.18703 \\
$\mathrm{H}$ & 0.08503 & 5.68584 & 2.01064 \\
$\mathrm{H}$ & 0.84866 & 6.62247 & -0.18136 \\
$\mathrm{H}$ & 2.12816 & 4.84207 & -1.50017 \\
$\mathrm{H}$ & 1.24624 & 3.56794 & -2.34699 \\
$\mathrm{H}$ & -0.91446 & 6.56538 & -0.11373 \\
$\mathrm{H}$ & -1.12685 & 3.50160 & 2.07166 \\
$\mathrm{H}$ & 0.12592 & 1.72950 & 0.73670 \\
$\mathrm{H}$ & -0.07602 & 5.66242 & -2.29774 \\
$\mathrm{H}$ & -2.07561 & 4.73340 & 1.24629 \\
$\mathrm{H}$ & 0.07688 & 1.73515 & -1.02286 \\
$\mathrm{C}$ & -2.48466 & 2.33463 & -0.03904 \\
$\mathrm{H}$ & -1.30590 & 3.49187 & -2.24944 \\
$\mathrm{H}$ & -2.17971 & 4.73066 & -1.35365 \\
$\mathrm{C}$ & -2.44043 & 0.93375 & -0.05877 \\
$\mathrm{C}$ & -3.75713 & 2.92948 & 0.02151 \\
$\mathrm{C}$ & -4.92194 & 2.16775 & 0.05683 \\
$\mathrm{C}$ & -3.60661 & 0.16490 & -0.02232 \\
$\mathrm{C}$ & -4.86904 & 0.76588 & 0.03446 \\
$\mathrm{H}$ & -3.84798 & 4.01220 & 0.04039 \\
$\mathrm{H}$ & -5.88827 & 2.66339 & 0.10195 \\
$\mathrm{H}$ & -1.48848 & 0.41655 & -0.10690 \\
$\mathrm{H}$ & -3.53316 & -0.91945 & -0.04116 \\
& & &
\end{tabular}




\begin{tabular}{lccc}
$\mathrm{C}$ & -6.12304 & -0.05204 & 0.06635 \\
$\mathrm{C}$ & -6.84702 & -0.27796 & -1.12509 \\
$\mathrm{C}$ & -6.58775 & -0.58907 & 1.28690 \\
$\mathrm{C}$ & -8.07110 & -1.04857 & -1.09336 \\
$\mathrm{C}$ & -6.40088 & 0.23479 & -2.38673 \\
$\mathrm{C}$ & -7.80566 & -1.36970 & 1.31682 \\
$\mathrm{C}$ & -8.53510 & -1.58594 & 0.12722 \\
$\mathrm{C}$ & -8.78565 & -1.23761 & -2.32117 \\
$\mathrm{C}$ & -7.11223 & 0.02104 & -3.53708 \\
$\mathrm{C}$ & -8.32489 & -0.72149 & -3.50288 \\
$\mathrm{C}$ & -5.88710 & -0.37806 & 2.51912 \\
$\mathrm{H}$ & -5.47715 & 0.80184 & -2.41692 \\
$\mathrm{C}$ & -9.80280 & -2.38376 & 0.16110 \\
$\mathrm{H}$ & -9.70997 & -1.80418 & -2.30040 \\
$\mathrm{H}$ & -6.75129 & 0.41997 & -4.48139 \\
$\mathrm{H}$ & -8.88584 & -0.87965 & -4.42020 \\
$\mathrm{H}$ & -4.98011 & 0.21597 & 2.50304 \\
$\mathrm{C}$ & -6.33920 & -0.90767 & 3.69816 \\
$\mathrm{C}$ & -7.52809 & -1.68806 & 3.72488 \\
$\mathrm{H}$ & -5.78876 & -0.73304 & 4.61885 \\
$\mathrm{C}$ & -8.23500 & -1.90913 & 2.57322 \\
$\mathrm{H}$ & -7.87505 & -2.10919 & 4.66478 \\
$\mathrm{H}$ & -9.14073 & -2.50474 & 2.59840 \\
$\mathrm{C}$ & -11.01618 & -1.78380 & 0.53097 \\
$\mathrm{C}$ & -9.80374 & -3.74616 & -0.17474 \\
$\mathrm{C}$ & -10.98523 & -4.48871 & -0.14145 \\
$\mathrm{C}$ & -12.19816 & -2.52535 & 0.56451 \\
$\mathrm{C}$ & -12.18602 & -3.88028 & 0.22851 \\
$\mathrm{H}$ & -8.86997 & -4.22233 & -0.46192 \\
$\mathrm{H}$ & -10.96631 & -5.54337 & -0.40381 \\
$\mathrm{H}$ & -11.02810 & -0.72902 & 0.79252 \\
$\mathrm{H}$ & -13.12877 & -2.04320 & 0.85262 \\
$\mathrm{H}$ & -13.10610 & -4.45809 & 0.25472 \\
$\mathrm{C}$ & 2.85166 & 1.51602 & 0.87773 \\
$\mathrm{C}$ & 3.55790 & 2.43310 & -1.21598 \\
$\mathrm{C}$ & 4.69712 & 1.62516 & -1.18428 \\
$\mathrm{C}$ & 3.98501 & 0.70754 & 0.91328 \\
$\mathrm{C}$ & 4.93293 & 0.74776 & -0.12010 \\
$\mathrm{H}$ & 3.42327 & 3.09547 & -2.06389 \\
$\mathrm{H}$ & 5.41377 & 1.67793 & -1.99988 \\
& & & \\
\hline
\end{tabular}

\begin{tabular}{|c|c|c|c|}
\hline $\mathrm{H}$ & 2.14740 & 1.45327 & 1.70302 \\
\hline $\mathrm{H}$ & 4.14139 & 0.03611 & 1.75364 \\
\hline $\mathrm{C}$ & 6.15454 & -0.11780 & -0.08147 \\
\hline $\mathrm{C}$ & 7.35781 & 0.38302 & 0.46197 \\
\hline $\mathrm{C}$ & 6.10241 & -1.43553 & -0.58591 \\
\hline $\mathrm{C}$ & 8.53780 & -0.45321 & 0.49924 \\
\hline $\mathrm{C}$ & 7.45129 & 1.71220 & 0.98940 \\
\hline $\mathrm{C}$ & 7.28064 & -2.27396 & -0.54305 \\
\hline $\mathrm{C}$ & 8.48427 & -1.77198 & -0.00215 \\
\hline $\mathrm{C}$ & 9.74138 & 0.09550 & 1.05063 \\
\hline $\mathrm{C}$ & 8.62259 & 2.19310 & 1.51086 \\
\hline $\mathrm{C}$ & 9.78498 & 1.37371 & 1.53980 \\
\hline $\mathrm{C}$ & 4.90284 & -1.97934 & -1.15055 \\
\hline $\mathrm{H}$ & 6.56754 & 2.34017 & 0.96832 \\
\hline $\mathrm{C}$ & 7.18319 & -3.60831 & -1.05654 \\
\hline $\mathrm{C}$ & 9.70568 & -2.63893 & 0.04085 \\
\hline $\mathrm{H}$ & 10.62976 & -0.52584 & 1.07502 \\
\hline $\mathrm{H}$ & 8.66723 & 3.20514 & 1.90450 \\
\hline $\mathrm{H}$ & 10.71047 & 1.76624 & 1.95275 \\
\hline $\mathrm{H}$ & 4.01955 & -1.35155 & -1.19062 \\
\hline $\mathrm{C}$ & 4.85731 & -3.25987 & -1.63343 \\
\hline $\mathrm{H}$ & 3.93502 & -3.64860 & -2.05701 \\
\hline $\mathrm{C}$ & 6.01344 & -4.08713 & -1.58335 \\
\hline $\mathrm{H}$ & 8.06232 & -4.24213 & -1.02025 \\
\hline $\mathrm{H}$ & 5.96549 & -5.10327 & -1.96580 \\
\hline $\mathrm{C}$ & 10.56878 & -2.71650 & -1.06264 \\
\hline $\mathrm{C}$ & 10.01208 & -3.38944 & 1.18606 \\
\hline $\mathrm{C}$ & 11.15088 & -4.19562 & 1.22737 \\
\hline $\mathrm{C}$ & 11.70809 & -3.52198 & -1.02219 \\
\hline $\mathrm{C}$ & 12.00237 & -4.26383 & 0.12323 \\
\hline $\mathrm{H}$ & 9.35055 & -3.33732 & 2.04675 \\
\hline $\mathrm{H}$ & 11.37197 & -4.77048 & 2.12301 \\
\hline $\mathrm{H}$ & 10.34207 & -2.13939 & -1.95521 \\
\hline $\mathrm{H}$ & 12.36585 & -3.56896 & -1.88628 \\
\hline $\mathrm{H}$ & 12.88930 & -4.89110 & 0.15517 \\
\hline
\end{tabular}

(EOF) 